\title{
Effect of Structural Phase Transition on Charge-Carrier Lifetimes and Defects in $\mathrm{CH}_{3} \mathrm{NH}_{3} \mathrm{Snl}_{3}$ Perovskite
}

\author{
Elizabeth S. Parrott, Rebecca L. Milot, Thomas Stergiopoulos, Henry J. Snaith, Michael B. Johnston, \\ and Laura M. Herz*
}

Department of Physics, University of Oxford, Clarendon Laboratory, Parks Road, Oxford OX1 3PU, United Kingdom

\section{Supporting Information}

ABSTRACT: Methylammonium tin triiodide $\left(\mathrm{MASnI}_{3}\right)$ has been successfully employed in leadfree perovskite solar cells, but overall power-conversion efficiencies are still significantly lower than for lead-based perovskites. Here we present photoluminescence (PL) spectra and timeresolved PL from 8 to $295 \mathrm{~K}$ and find a marked improvement in carrier lifetime and a substantial reduction in PL line width below $\sim 110 \mathrm{~K}$, indicating that the cause of the hindered performance is activated at the orthorhombic to tetragonal phase transition. Our measurements therefore suggest that targeted structural change may be capable of tailoring the relative energy level alignment of defects (e.g., tin vacancies) to reduce the background dopant density and improve charge extraction. In addition, we observe for the first time an above-gap emission feature that may arise from higher-lying interband transitions, raising the prospect of excess energy harvesting.

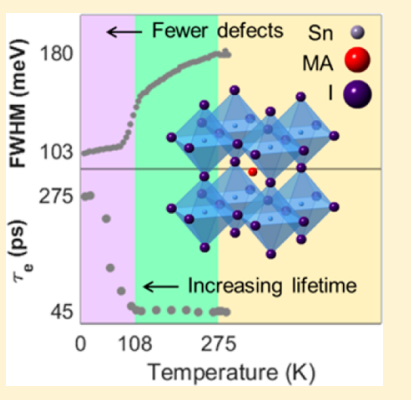

rganic-metal-halide perovskites have emerged as a new class of solution-processable direct-bandgap semiconductors for photovoltaic device applications. ${ }^{1-5}$ Solar cells with lead-based perovskites have now reached remarkable power conversion efficiencies ${ }^{6,7}$ (PCEs) of over $20 \%$, owing to long charge-carrier diffusion lengths ${ }^{8,9}$ and benign defect chemistry; $^{10,11}$ however, there have been some environmental concerns about using these materials in large-scale applications because of the possibility of lead contamination due to leakage from damaged solar panels. ${ }^{12-15}$ As a lead-free alternative, tinbased perovskites have been implemented in solar cells. ${ }^{16-21}$ For methylammonium tin triiodide $\left(\mathrm{MASnI}_{3}\right)$ an open-circuit voltage of $0.88 \mathrm{~V}$ has been reported, which is high compared with the band gap of around 1.2 to $1.3 \mathrm{eV}^{16}$ however, the short-circuit currents and fill factors remain low with maximum reported values of $21 \mathrm{~mA} \mathrm{~cm}{ }^{-218}$ and $0.48,{ }^{17}$ respectively, and the record PCE stands at $6 \%,{ }^{16}$ far below that for lead-based perovskite photovoltaics. It has been suggested that the low performance of tin-based perovskites is caused by the low formation energy of tin vacancies, ${ }^{20,22,23}$ resulting in excessive p-doping. Several studies have shown that both $\mathrm{MASnI}_{3}$ and $\mathrm{CsSnI}_{3}$ can become spontaneously hole-doped during crystal formation with dark carrier densities ranging from $10^{14}$ to $10^{19}$ $\mathrm{cm}^{-3}$ depending on preparation method. ${ }^{17,18,21,22,24,25}$ Such doping may lead to strong monomolecular charge-carrier decay components $^{16,21}$ from electron recombination with the large background hole density, which may limit the carrier diffusion and extraction. ${ }^{16,19,21,24}$ First-principles calculations for $\mathrm{CsSnI}_{3}$ have suggested that tin vacancies, and indeed all acceptor-type defects, have energy levels close to the valence band edge and are therefore easily ionized, thus p-doping the material. ${ }^{23}$

Despite the importance of such tin vacancies, experimental studies of their nature and their effect on the optoelectronic properties are relatively scarce. Here we have investigated such links by monitoring the photoluminescence (PL) and absorption spectra and the PL lifetime of $\mathrm{MASnI}_{3}$ as a function of temperature from 8 to $295 \mathrm{~K}$. We find that at room temperature the charge lifetime is short, and the emission line width is severely broadened in accordance with the presence of a sizable density of defects that act as hole donors. As the temperature is reduced below the phase transition into the orthorhombic phase near $110 \mathrm{~K}$, a significant line narrowing and recovery of charge-carrier lifetimes is observed, and an additional emission peak originating from higher energy transitions appears. These abrupt changes coincide with a previously reported structural phase transition ${ }^{24}$ and a decrease in hole concentration, ${ }^{26}$ which indicates that crystal structure, hole doping, disorder, and carrier lifetime are intrinsically linked. Such improved understanding of links between defect chemistry and crystal structure may therefore highlight routes toward further optimization of tin-based perovskites.

We deposited $\mathrm{MASnI}_{3}$ perovskite on z-cut quartz disks by spin-coating using methylammonium iodide and tin(II) acetate precursor solutions (see Supporting Information). Because of the known instability of tin perovskites in air, we prepared the samples and mounted them into a cryostat inside a nitrogenfilled glovebox. We carried out measurements under vacuum, with samples for PL measurements never being exposed to air and samples for absorption measurements being exposed to air for less than a minute during transfer. Measurements were taken starting from room temperature $(295 \mathrm{~K})$ and cooling with liquid helium to $8 \mathrm{~K}$. We observed no change to the original room-temperature properties once the sample was

Received: February 12, 2016

Accepted: March 18, 2016

Published: March 18, 2016 
returned to room temperature after cooling, showing that no permanent damage had occurred.

We first examine the general shape of the absorption and emission spectra of $\mathrm{MASnI}_{3}$ as a function of temperature. Figure 1a shows a map of the PL intensity as a function of

(a)

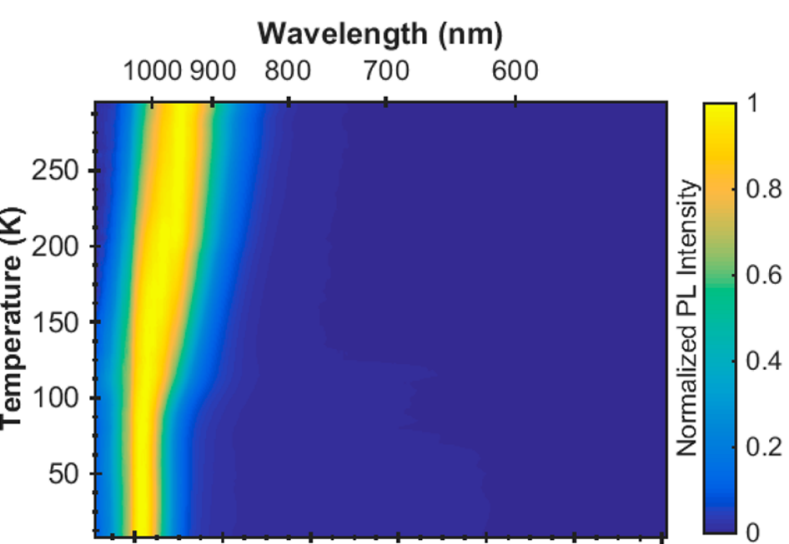

(b)

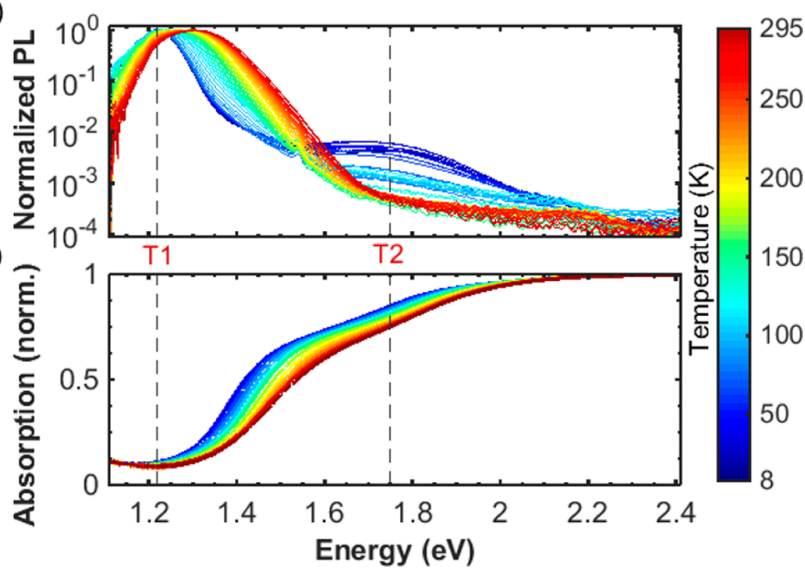

Figure 1. (a) Color map of photoluminescence intensity as a function of temperature, normalized to the peak intensity at each temperature. We excited the sample with a pulsed laser at $400 \mathrm{~nm}$ with a fluence of $3 \mathrm{~nJ} \mathrm{~cm}^{-2}$ and collected spectra at intervals of $5 \mathrm{~K}$, cooling the sample from room temperature. (b) Photoluminescence spectra on a semilog scale and (c) absorption spectra (without reflection correction) from high temperature (red) to low temperature (blue) in the range 8-295 $\mathrm{K}$. Dashed vertical lines mark the two peak PL energies at $8 \mathrm{~K}$.

temperature between 8 and $295 \mathrm{~K}$. The peak emission at room temperature is found to occur at $1.3 \mathrm{eV}$, near the range of theoretical calculations ${ }^{27}$ and previous measurements ${ }^{16-18,25}$ of the band gap energy. As the temperature is decreased, the PL peak shifts to lower energy, similar to previously reported band gap trends in $\mathrm{CsSnI}_{3}{ }^{28}$ and $\mathrm{MAPbI}_{3}{ }^{29}$ This gradual red shift is mirrored in the absorption spectrum (Figure 1c) obtained from measuring the optical transmission of $\mathrm{MASnI}_{3}$ using Fourier transform infrared (FTIR) spectroscopy. We observe a clear absorption onset corresponding to the transition at the band gap (T1), which is centered $\sim 200 \mathrm{meV}$ above the PL peak emission energy, suggesting a sizable Stokes shift.

$\mathrm{MASnI}_{3}$ is known to exhibit phase transitions at $275^{30,31}$ and $108 \mathrm{~K}^{31}$ when cooled (but $114 \mathrm{~K}$ when heated), transitioning from pseudocubic $(\alpha)$ at room temperature ${ }^{25}$ to tetragonal $(\beta)$ to orthorhombic $(\gamma)$ at low temperature. Interestingly, we observe no discontinuity in absorption or PL energy with temperature at the phase transitions, which suggests that the band-edge states do not substantially change here. This behavior vastly differs from that at the $\beta$ to $\gamma$ phase transition $(160 \mathrm{~K})$ in $\mathrm{MAPbI}_{3}$, which results in a $100 \mathrm{meV}$ shift in bandgap energy; ${ }^{29,32}$ however, as we show later, the phase transition in $\mathrm{MASnI}_{3}$ at $\sim 110 \mathrm{~K}$ is predominantly associated with changes in impurity-induced disorder and charge-carrier recombination dynamics.

Before we analyze the effect of disorder and impurities on the optoelectronic properties of $\mathrm{MASnI}_{3}$ we first highlight a peculiar occurrence, which is the appearance of a secondary, higher-energy absorption and emission feature near $1.75 \mathrm{eV}$, marked as T2 in Figure 1b. This second PL peak is generally very weak (note the logarithmic scale); it emerges at $110 \mathrm{~K}$ and grows in intensity with respect to the main peak as the temperature is decreased further, reaching an intensity of one hundredth of that of the main peak at the lowest temperature. In contrast, a matching absorption feature for $\mathrm{T} 2$ is seen over the entire temperature range (Figure 1c), which red shifts in the same way as the band edge (Figure 2a). Therefore, this

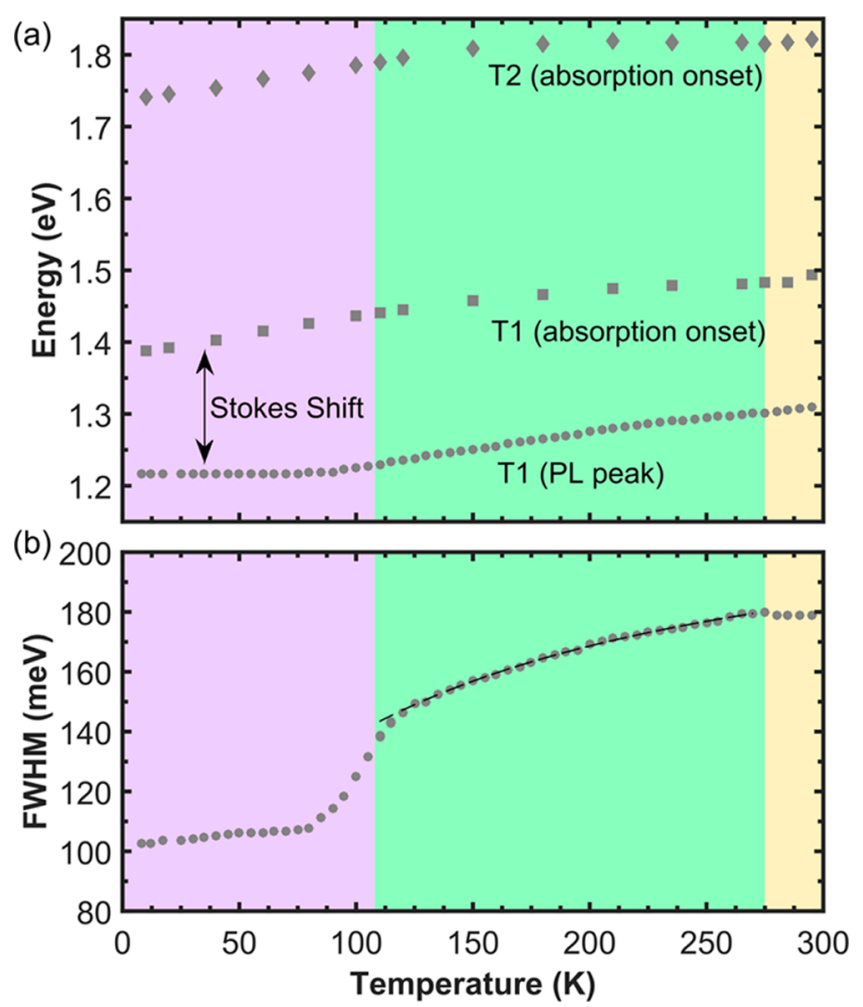

Figure 2. (a) Energies of the two transitions apparent in the absorption and emission spectra of $\mathrm{MASnI}_{3}, \mathrm{~T} 1$ (absorption, squares; emission circles) and T2 (absorption, diamonds). Emission energies were taken from PL peaks, and absorption energies were taken from the midpoint of the broadened absorption rise, as determined by finding the maximum gradient. Note that the midpoint is not exactly equal to the band gap but gives a direct indication of how the band gap shifts with temperature. (b) Line width of PL peak T1, determined by fitting a Gaussian to the PL spectra (see Figure S2). The dashed black line shows the equation $\Gamma(\mathrm{T})=\gamma_{\text {imp }} \mathrm{e}^{-E_{\mathrm{b}} / k T}+\Gamma_{0}$ with $E_{\mathrm{b}}=10 \mathrm{meV}$, $\gamma_{\text {imp }}=119 \mathrm{meV}$, and $\Gamma_{0}=103 \mathrm{meV}$.

higher-energy transition T2 appears to be intrinsic to the band structure of $\mathrm{MASnI}_{3}$, persisting over the entire temperature range but only yielding efficient emission in the orthorhombic phase at low temperature.

We note that a similar secondary onset is also seen in the optical absorption spectrum of $\mathrm{MAPbI}_{3}$ (at $2.6 \mathrm{eV}$ or $480 \mathrm{~nm}$ ) 
and found to coincide with a photobleach signal in transient absorption spectra, ${ }^{33-35}$ but luminescence at this wavelength has never been reported. This transition has been variously attributed to an additional valence band, ${ }^{33}$ to a different valley in the reciprocal lattice, ${ }^{36}$ or to a charge-transfer state. ${ }^{34} \mathrm{~A}$ further photobleach signal in $\mathrm{MAPbI}_{3}$ is sometimes seen at $\sim 2.43 \mathrm{eV}(510 \mathrm{~nm})$ and attributed to excess $\mathrm{PbI}_{2} ;{ }^{37}$ however, $\mathrm{SnI}_{2}$ has a band gap of $2.57 \mathrm{eV}$ (at $\left.8 \mathrm{~K}\right),{ }^{38}$ far from the peak we observe at $1.75 \mathrm{eV}$; therefore, $\mathrm{T} 2$ cannot be caused by excess $\mathrm{SnI}_{2}$.

We therefore attribute the origin of the emission observed at around 1.7 to $1.8 \mathrm{eV}$ to additional transitions associated with the electronic band structure of $\mathrm{MASnI}_{3}$. Here the most likely candidate is a transition between the highest-lying valence band maximum (VB1) and the minimum of the split-off conduction band (CB2) at the zone center (see Figure S5). Theoretical calculations predict that spin orbit coupling is important in the band structure of both lead and tin perovskites, resulting in two conduction bands, $\mathrm{CB} 1$ and $\mathrm{CB} 2$, split by the energy of the spin-orbit interaction $E_{\mathrm{SOC}}{ }^{39}$ For $\mathrm{MASnI}_{3}, E_{\mathrm{SOC}}=0.4 \mathrm{eV}$, compared with $1.5 \mathrm{eV}$ for $\mathrm{MAPbI}_{3}{ }^{40}$ Two optically allowed transitions are therefore associated with these bands-a strong transition from VB1 to $\mathrm{CB} 1$, associated with band gap energy $E_{\mathrm{g}}=1.3 \mathrm{eV}$, and another weak transition from VB1 to CB2 with energy $E=E_{\mathrm{g}}+E_{\mathrm{SOC}}=1.7 \mathrm{eV} .^{39,40}$ These theoretical predictions correspond to the energies of the transitions we observe, making this a likely explanation. Alternatively, these transitions could arise from different electronic bands, or different points in the Brillouin zone (Figure S5); however, the latter would require exceptionally slow intraband carrier thermalization, which is unlikely. We note that the proposed mechanism of emission from charge-carrier recombination involving split-off bands has also been observed in the direct semiconductor GaAs, ${ }^{41,42}$ which highlights the similarity of these perovskites with fully inorganic semiconducting materials.

We continue to examine the optoelectronic properties of $\mathrm{MASnI}_{3}$ by investigating changes in the emission line width with temperature, which reveals the influence of disorder and impurities. The PL line width $(\Gamma)$ was determined from the full width at half-maximum (FWHM) of the emission spectrum and is shown in Figure $2 \mathrm{~b}$ as a function of temperature. We find $\Gamma$ for $\mathrm{MASnI}_{3}$ to be rather large, with a value of $180 \mathrm{meV}$ at room temperature compared with $103 \mathrm{meV}$ for $\mathrm{MAPbI}_{3}{ }^{43}$ and $50 \mathrm{meV}$ for $\mathrm{CsSnI}_{3}{ }^{44}$ In general, both homogeneous line width broadening (typically from phonons) and inhomogeneous broadening (from impurities and energetic disorder) may contribute to the overall width of the emission spectrum. The resulting emission line width is hence described by the following equation ${ }^{45}$

$$
\Gamma(T)=\gamma_{\mathrm{ac}} T+\gamma_{\mathrm{LO}} /\left(\mathrm{e}^{E_{\mathrm{LO}} / k T}-1\right)+\gamma_{\mathrm{imp}} \mathrm{e}^{-E_{\mathrm{b}} / k T}+\Gamma_{0}
$$

Here the terms (in order of appearance) arise from scattering with acoustic phonons (coupling strength $\gamma_{\mathrm{ac}}$ ), optical phonons of energy $E_{\mathrm{LO}}$ (coupling $\gamma_{\mathrm{LO}}$ ), donor/acceptor impurities with ionization energy $E_{\mathrm{b}}$ (coupling $\gamma_{\text {imp }}$ ), and a temperatureindependent broadening term $\Gamma_{0}$ arising, for example, from energetic disorder. In the high-temperature range the phonon terms increase linearly with $T$ (see Figure S6 for example curves), which is contrary to the sublinear dependence of the line width on temperature we observe. Such sublinear behavior is, however, accurately described by the functional form of the ionizable impurity term in the above equation, suggesting that scattering of charge-carriers from ionized impurities such as tin vacancies is the predominant contribution to line width broadening in $\mathrm{MASnI}_{3}$ above $110 \mathrm{~K}$. Because the impurity terms dominate, we neglect phonon contributions and fit the line width from 140 to $270 \mathrm{~K}$ (within the tetragonal phase), which gives a binding energy of $\sim 10 \mathrm{meV}$ (Figure 2), although the small-temperature range means that binding energies from 10 to $20 \mathrm{meV}$ give reasonable fits (Figure S7); however, this functional form alone cannot account for the surprisingly abrupt line width narrowing below $110 \mathrm{~K}$. We propose that because the line width in this material is dominated by impurities, the sudden change must be associated with a sharp decrease in number or change in binding energy of the defects below the phase transition. As previously mentioned, the most easily formed point defects in tin iodide perovskites are tin vacancies. ${ }^{22,23}$ Removing a tin atom can contribute two holes to the valence band ${ }^{22}$ if the resulting defect energy level lies close to the valence band, as predicted. ${ }^{23}$ At sufficiently low temperature $\left(k T<E_{\mathrm{b}}\right)$, however, holes can potentially become localized to their acceptor ions (such as tin vacancies), making the defect charge-neutral and lowering the interaction with charge-carriers, as described in the above model. Hence the emission line width is expected to narrow as dopant charge carriers localize. The abrupt change at the phase transition may indicate that a structural change makes it favorable for the holes to become localized, resulting in a sudden decrease in the density of holes in the valence band. We note that this explanation agrees well with the increase in electrical resistivity that has previously been observed ${ }^{24,26}$ when $\mathrm{MASnI}_{3}$ was lowered below the transition into the orthorhombic phase at $110 \mathrm{~K}$. The large change in line width at the phase transition, despite the absence of strong changes in band gap energy, is surprising; however, it suggests that relatively small changes in structure can suffice to suppress excess dopant carrier density in tin halide perovskites.

The temperature-independent inhomogeneous line width value $\left(\Gamma_{0}=103 \mathrm{meV}\right)$ approached at very low temperature reflects the sizable disorder remaining in this phase, despite the improvement at the $110 \mathrm{~K}$ transition. The large contribution to the emission broadening in $\mathrm{MASnI}_{3}$, from both ionizable defects $\left(\gamma_{\text {imp }}\right)$ and temperature-independent disorder $\left(\Gamma_{0}\right)$, is in stark contrast with $\mathrm{MAPbI}_{3}$ for which the broadening is thought to be almost entirely homogeneous at room temperature. ${ }^{43}$ Similar conclusions can be made when comparing the Stokes shifts between the absorption onset and the emission peak observed for these two materials. Here we find a large Stokes shift of almost $200 \mathrm{meV}$ for $\mathrm{MASnI}_{3}$ independent of temperature (Figure 2a), whereas for $\mathrm{MAPbI}_{3}$, Stokes shifts of at most $10 \mathrm{meV}$ have been reported, ${ }^{29}$ in accordance with significantly higher energetic disorder in the tin perovskite. Such contrast in defect densities has also been reported between copper indium gallium sulfide (CIGS) and copper zinc tin sulfide (CZTS), where defects in CZTS result in tail states that protrude into the band gap. ${ }^{46,47}$ Tail states cause the peak PL energy to be considerably red-shifted from the absorption onset because carriers tend to thermalize into the tail states, which is typically accompanied by an undesirable lowering of charge-carrier mobility.

Finally, we investigated the effect of temperature-dependent doping and disorder on the charge-carrier lifetime. We measured time-resolved PL transients using time-correlated single photon counting (TCSPC) after excitation with laser pulses of $400 \mathrm{~nm}$ wavelength, as shown in Figure 3a. Because 

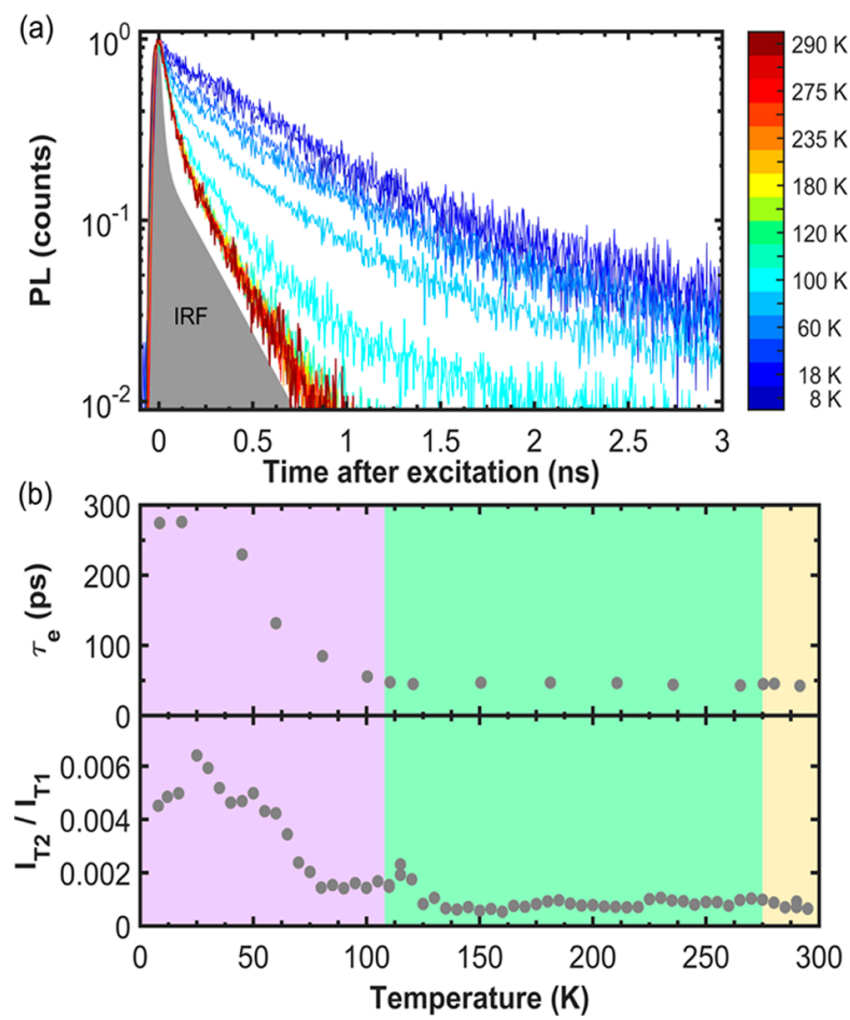

Figure 3. (a) Time-resolved photoluminescence (PL) as a function of time after excitation, from high temperature (red) to low temperature (blue). The instrument response function is shown by the shaded gray area. (b) Top: PL lifetime determined as the time to reach 1/e of the maximum intensity. Bottom: relative intensity of the second transition (T2) intensity in comparison with that originating from the band gap (T1).

the PL lifetime is very short, it is important to take account of the instrument response function (IRF) shown by the gray shaded area, which was obtained by measuring the response of the detector to laser pulses at $990 \mathrm{~nm}$ (near the detection wavelength). Because a single functional form for the decay, convoluted with the IRF, could not fit the entire temperature range satisfactorily we chose to use the $1 / \mathrm{e}$ lifetime to quantitatively compare the decays. We smoothed the data and IRF and deconvoluted the two using a fast Fourier transform algorithm to obtain a corrected decay curve (see Figure S1). Using this method, we determined the time $\tau_{\mathrm{e}}$ taken for the PL to decay to $1 / \mathrm{e}$ of its initial value, shown in Figure $3 \mathrm{~b}$ as a function of temperature. The PL lifetime changes very little at temperatures above $110 \mathrm{~K}$, remaining fixed at $\sim 45 \mathrm{ps}$, which is considerably shorter than lifetimes for $\mathrm{MAPbI}_{3}$ (typically on the order of $100-1000 \mathrm{~ns}) .{ }^{48}$ The short carrier lifetime at room temperature is problematic for planar device architectures, which require a long diffusion length for carrier extraction, although if the carrier mobilities are high enough, planar architectures will still be achievable. ${ }^{16,49}$ In addition, we find the shape of the room-temperature PL transients to be independent of excitation fluence over a large measured range $\left(0.3-300 \mathrm{~nJ} \mathrm{~cm}^{-2}\right)$, as shown in Figure S4. This behavior implies that monomolecular recombination is heavily dominant, rather than bimolecular electron-hole recombination that would result in fluence-dependent dynamics. ${ }^{11}$ Monomolecular recombination is expected to dominate when there is a high level of defects that act as recombination centers ${ }^{11}$ or there is a high background carrier concentration such that the photoexcited carrier concentration has little influence on the dynamics. ${ }^{16}$ Hence the observed fast carrier recombination dynamics in this temperature region is compatible with the broad emission line width observed and the presence of a large tin vacancy density.

Below $\sim 110 \mathrm{~K}$ the lifetime increases steeply, reaching a value of $\sim 275$ ps at $8 \mathrm{~K}$. Furthermore, the dynamics become dependent on excitation fluence here (see Figure S4) for the same investigated range, suggesting the background carrier concentration is now lower. This explanation correlates well with the observed steep decline in emission line width previously discussed. If, as we have already suggested, there is a change in the energy levels of the acceptor ions such that they are elevated further above the valence band maximum, it will become suddenly more favorable for the holes to be localized to these energy levels as the temperature is decreased, causing the hole density to drop. It is worth noting that a rise in lifetime at low temperature is also seen in CIGS and CZTS, with CZTS showing a thousand-fold increase. ${ }^{47}$ This is attributed to spatial electrostatic variations due to high levels of disorder or defects, which confines the carriers in different locations, providing a barrier to recombination that is overcome thermally as the temperature is increased. Hence charge-carrier lifetimes can sensitively depend on both recombination with a density of impurity-induced background carriers and the energetic disorder associated with such impurities.

Interestingly, this rise in carrier lifetime below the phasetransition temperature concurs with the observed increase in intensity of the secondary PL peak T2 (Figure 3b), alluding to a common cause. This could simply be due to the interplay between the rate constants for recombination at the transitions $\mathrm{T} 1$ and $\mathrm{T} 2$. While T1 may be mostly dominated by electron recombination with the sizable dark hole density, T2 could be largely influenced by phonon-mediated interband transitions, for example, between multiple conduction (or valence) bands. As a result, the relative rates may therefore be affected differently at the phase transition, imparting the observed changes in relative emission intensity (Figure $3 \mathrm{~b}$ ).

Taken together, these observations point to a significant change between the high- and low-temperature regimes, which is induced by the phase transition at $108 \mathrm{~K}$. We suggest that all of these features, the increase in PL lifetime, the drop in FWHM, the appearance of PL from a secondary transition, and the previously observed decrease in hole concentration, ${ }^{26}$ are related to the presence of defects in the system (likely tin vacancies that have a low formation energy ${ }^{22}$ ). These defects scatter carriers, giving the PL a broad line width, and also pdope the material, resulting in a large background hole density, which, in turn, leads to rapid charge-carrier recombination. Our observations suggest that upon the transition into the orthorhombic phase, the relative alignment of the defects' energy level must change subtly with respect to the valence band edge. This change in energy level results in background holes becoming localized to defects that reduce their contribution to carrier scattering (emission line width decreases) and also reduce the p-doping (carrier lifetime increases). Further reduction in temperature freezes out more background holes such that the lifetime increases further. An understanding of the precise mechanisms inducing these effects is important because it may hold the clue to achieving similar effects at room temperature. Scattering between charge-carriers and impurities leads to reduced charge-carrier mobilities, while 
charge-recombination with background holes reduces chargecarrier lifetimes. In combination, these effects account for the significantly lowered charge-carrier diffusion length of only a few tens of nanometers observed for $\mathrm{MASnI}_{3}$ at room temperature. ${ }^{16}$ Our measurements indicate that while the absorption onset energy hardly changes at the phase transition near $110 \mathrm{~K}$, the relative energetic alignment of defect levels with respect to the valence band edge is significantly altered. An understanding of how this favorable alignment is induced by the structural changes could potentially allow for such effects to be replicated at room temperature through compositional changes.

In summary, we have evaluated the optoelectronic properties of $\mathrm{MASnI}_{3}$ as a function of temperature to reveal the influence of impurities on charge-carrier scattering and recombination lifetimes. We find an abrupt improvement as the temperature is lowered into the orthorhombic phase below $108 \mathrm{~K}$, where emission line widths narrow and lifetimes increase significantly. We suggest that this effect is caused by a change to the energy level alignment of the tin vacancies with respect to the valence band maximum, leading to a reduction in background dopant density. First-principle calculations, that relate these effects to the structural changes anticipated at the phase transition, could hold the key to further material improvement. Because the perovskite structure allows for a range of organic/inorganic cations and halide anions to be combined with $\mathrm{Sn}^{2+}$, a material combination may be found that replicates the favorable change observed at the phase transition at room temperature, allowing for low background hole densities. In addition, we have observed a weak but distinct above-gap luminescence peak that coincides with the energy of a secondary absorption onset that is seen in similar form in many other hybrid metal halide perovskites. We propose that this emission originates from charge-carrier recombination involving a higher-lying band, for example, split off by spin-orbit coupling, similar to what has been observed for GaAs. The observation of such emission suggests that a certain fraction of charges exists for an appreciable time in a higher-energy state. If such excess energy could be harnessed, this could offer a further efficiency boost and provide a means for exceeding the Shockley-Queisser limit.

\section{ASSOCIATED CONTENT}

\section{S Supporting Information}

The Supporting Information is available free of charge on the ACS Publications website at DOI: 10.1021/acs.jpclett.6b00322.

Experimental methods, data fitting and analysis, fluencedependent PL decay curves, schematics of recombination, and sample emission broadening curves. (PDF)

\section{AUTHOR INFORMATION}

\section{Corresponding Author}

*E-mail: laura.herz@physics.ox.ac.uk.

\section{Notes}

The authors declare no competing financial interest.

\section{ACKNOWLEDGMENTS}

We acknowledge the Engineering and Physical Sciences Research Council (EPSRC) for financial support. E.S.P thanks the EPSRC Centre for Doctoral Training in New and Sustainable Photovoltaics (CDT-PV) for support through a doctoral studentship. T.S. acknowledges financial support by a
Marie Curie Intra European Fellowship (MESO-SUPERCELLS, PIEG-GA-2012-327015) within the 7th European Community Framework Programme.

\section{REFERENCES}

(1) Kojima, A.; Teshima, K.; Shirai, Y.; Miyasaka, T. Organometal Halide Perovskites as Visible-Light Sensitizers for Photovoltaic Cells. J. Am. Chem. Soc. 2009, 131, 6050-6051.

(2) Lee, M. M.; Teuscher, J.; Miyasaka, T.; Murakami, T. N.; Snaith, H. J. Efficient Hybrid Solar Cells Based on Meso-Superstructured Organometal Halide Perovskites. Science 2012, 338, 643-648.

(3) Kim, H.-S.; Lee, C.-R.; Im, J.-H.; Lee, K.-B.; Moehl, T.; Marchioro, A.; Moon, S.-J.; Humphry-Baker, R.; Yum, J.-H.; Moser, J. E.; et al. Lead Iodide Perovskite Sensitized All-Solid-State Submicron Thin Film Mesoscopic Solar Cell with Efficiency Exceeding 9\%. Sci. Rep. 2012, 2, 591.

(4) Liu, M.; Johnston, M. B.; Snaith, H. J. Efficient Planar Heterojunction Perovskite Solar Cells by Vapour Deposition. Nature 2013, 501, 395-398.

(5) Green, M. A.; Ho-Baillie, A.; Snaith, H. J. The Emergence of Perovskite Solar Cells. Nat. Photonics 2014, 8, 506-514.

(6) Yang, W. S.; Noh, J. H.; Jeon, N. J.; Kim, Y. C.; Ryu, S.; Seo, J.; Seok, S., Il. High-Performance Photovoltaic Perovskite Layers Fabricated through Intramolecular Exchange. Science 2015, 348, 1234-1237.

(7) Bi, D.; Tress, W.; Dar, M. I.; Gao, P.; Luo, J.; Renevier, C.; Schenk, K.; Abate, A.; Giordano, F.; Correa Baena, J.-P.; et al. Efficient Luminescent Solar Cells Based on Tailored Mixed-Cation Perovskites. Sci. Adv. 2016, 2, e1501170.

(8) Stranks, S. D.; Eperon, G. E.; Grancini, G.; Menelaou, C.; Alcocer, M. J. P.; Leijtens, T.; Herz, L. M.; Petrozza, A.; Snaith, H. J. Electron-Hole Diffusion Lengths Exceeding 1 Micrometer in an Organometal Trihalide Perovskite Absorber. Science 2013, 342, 341344.

(9) Wehrenfennig, C.; Eperon, G. E.; Johnston, M. B.; Snaith, H. J.; Herz, L. M. High Charge Carrier Mobilities and Lifetimes in Organolead Trihalide Perovskites. Adv. Mater. 2014, 26, 1584-1589.

(10) Yin, W.-J.; Shi, T.; Yan, Y. Unusual Defect Physics in $\mathrm{CH}_{3} \mathrm{NH}_{3} \mathrm{PbI}_{3}$ Perovskite Solar Cell Absorber. Appl. Phys. Lett. 2014, 104, 063903.

(11) Herz, L. M. Charge Carrier Dynamics in Organic-Inorganic Metal Halide Perovskites. Annu. Rev. Phys. Chem. 2016, 67, DOI: 10.1146/annurev-physchem-040215-112222.

(12) Benmessaoud, I. R.; Mahul-Mellier, A.-L.; Horvath, E.; Maco, B.; Spina, M.; Lashuel, H.; Forro, L. Health Hazards of the Methylammonium Lead Iodide Based Perovskites: Cytotoxicity Studies. Toxicol. Res. 2016, 5, 407.

(13) Espinosa, N.; Serrano-Lujan, L.; Urbina, A.; Krebs, F. C. Solution and Vapour Deposited Lead Perovskite Solar Cells: Ecotoxicity from a Life Cycle Assessment Perspective. Sol. Energy Mater. Sol. Cells 2015, 137, 303-310.

(14) Zhang, J.; Gao, X.; Deng, Y.; Li, B.; Yuan, C. Life Cycle Assessment of Titania Perovskite Solar Cell Technology for Sustainable Design and Manufacturing. ChemSusChem 2015, 8, 3882-3891.

(15) Babayigit, A.; Duy Thanh, D.; Ethirajan, A.; Manca, J.; Muller, M.; Boyen, H.-G.; Conings, B. Assessing the Toxicity of Pb- and $\mathrm{Sn}-$ Based Perovskite Solar Cells in Model Organism Danio Rerio. Sci. Rep. 2016, 6, 18721.

(16) Noel, N. K.; Stranks, S. D.; Abate, A.; Wehrenfennig, C.; Guarnera, S.; Haghighirad, A.; Sadhanala, A.; Eperon, G. E.; Pathak, S. K.; Johnston, M. B.; et al. Lead-Free Organic-Inorganic Tin Halide Perovskites for Photovoltaic Applications. Energy Environ. Sci. 2014, 7, 3061-3068.

(17) Hao, F.; Stoumpos, C. C.; Cao, D. H.; Chang, R. P. H.; Kanatzidis, M. G. Lead-Free Solid-State Organic-inorganic Halide Perovskite Solar Cells. Nat. Photonics 2014, 8, 489-494. 
(18) Hao, F.; Stoumpos, C. C.; Guo, P.; Zhou, N.; Marks, T. J.; Chang, R. P. H.; Kanatzidis, M. G. Solvent-Mediated Crystallization of $\mathrm{CH}_{3} \mathrm{NH}_{3} \mathrm{SnI}_{3}$ Films for Heterojunction Depleted Perovskite Solar Cells. J. Am. Chem. Soc. 2015, 137, 11445-11452.

(19) Koh, T. M.; Krishnamoorthy, T.; Yantara, N.; Shi, C.; Leong, W. L.; Boix, P. P.; Grimsdale, A. C.; Mhaisalkar, S. G.; Mathews, N. Formamidinium Tin-Based Perovskite with Low $\mathrm{E}_{\mathrm{g}}$ for Photovoltaic Applications. J. Mater. Chem. A 2015, 3, 14996-15000.

(20) Marshall, K. P.; Walton, R. I.; Hatton, R. A. Tin Perovskite/ Fullerene Planar Layer Photovoltaics: Improving the Efficiency and Stability of Lead-Free Devices. J. Mater. Chem. A 2015, 3, 1163111640 .

(21) Kumar, M. H.; Dharani, S.; Leong, W. L.; Boix, P. P.; Prabhakar, R. R.; Baikie, T.; Shi, C.; Ding, H.; Ramesh, R.; Asta, M.; et al. LeadFree Halide Perovskite Solar Cells with High Photocurrents Realized Through Vacancy Modulation. Adv. Mater. 2014, 26, 7122-7127.

(22) Chung, I.; Song, J. H.; Im, J.; Androulakis, J.; Malliakas, C. D.; Li, H.; Freeman, A. J.; Kenney, J. T.; Kanatzidis, M. G. CsSnI ${ }_{3}$ : Semiconductor or Metal? High Electrical Conductivity and Strong Near-Infrared Photoluminescence from a Single Material. High Hole Mobility and Phase-Transitions. J. Am. Chem. Soc. 2012, 134, 85798587.

(23) Xu, P.; Chen, S.; Xiang, H.; Gong, X.; Wei, S. Influence of Defects and Synthesis Conditions on the Photovoltaic Performance of Perovskite Semiconductor CsSnI 3 . Chem. Mater. 2014, 26, 60686072.

(24) Takahashi, Y.; Hasegawa, H.; Takahashi, Y.; Inabe, T. Hall Mobility in Tin Iodide Perovskite $\mathrm{CH}_{3} \mathrm{NH}_{3} \mathrm{SnI}_{3}$ : Evidence for a Doped Semiconductor. J. Solid State Chem. 2013, 205, 39-43.

(25) Stoumpos, C. C.; Malliakas, C. D.; Kanatzidis, M. G. Semiconducting Tin and Lead Iodide Perovskites with Organic Cations: Phase Transitions, High Mobilities, and near-Infrared Photoluminescent Properties. Inorg. Chem. 2013, 52, 9019-9038.

(26) Mitzi, D.; Feild, C.; Schlesinger, Z.; Laibowitz, R. B. Transport, Optical and Magnetic Properties of the Conducting Halide Perovskite $\mathrm{CH}_{3} \mathrm{NH}_{3} \mathrm{SnI}_{3}$. J. Solid State Chem. 1995, 114, 159-163.

(27) Umari, P.; Mosconi, E.; De Angelis, F. Relativistic GW Calculations on $\mathrm{CH}_{3} \mathrm{NH}_{3} \mathrm{PbI}_{3}$ and $\mathrm{CH}_{3} \mathrm{NH}_{3} \mathrm{SnI}_{3}$ Perovskites for Solar Cell Applications. Sci. Rep. 2014, 4, 4467.

(28) Yu, C.; Chen, Z.; Wang, J. J.; Pfenninger, W.; Vockic, N.; Kenney, J. T.; Shum, K. Temperature Dependence of the Band Gap of Perovskite Semiconductor Compound $\mathrm{CsSnI}_{3}$. J. Appl. Phys. 2011, $110,063526$.

(29) Milot, R. L.; Eperon, G. E.; Snaith, H. J.; Johnston, M. B.; Herz, L. M. Temperature-Dependent Charge-Carrier Dynamics in $\mathrm{CH}_{3} \mathrm{NH}_{3} \mathrm{PbI}_{3}$ Perovskite Thin Films. Adv. Funct. Mater. 2015, 25, 6218-6227.

(30) Yamada, K.; Nakada, K.; Takeuchi, Y.; Nawa, K.; Yamane, Y. Tunable Perovskite Semiconductor $\mathrm{CH}_{3} \mathrm{NH}_{3} \mathrm{SnX}_{3}(\mathrm{X}: \mathrm{Cl}, \mathrm{Br}$, or I) Characterized by X-Ray and DTA. Bull. Chem. Soc. Jpn. 2011, 84, 926932.

(31) Takahashi, Y.; Obara, R.; Lin, Z.-Z.; Takahashi, Y.; Naito, T.; Inabe, T.; Ishibashi, S.; Terakura, K. Charge-Transport in Tin-Iodide Perovskite $\mathrm{CH}_{3} \mathrm{NH}_{3} \mathrm{SnI}_{3}$ : Origin of High Conductivity. Dalton Trans. 2011, 40, 5563-5568.

(32) Wehrenfennig, C.; Liu, M.; Snaith, H. J.; Johnston, M. B.; Herz, L. M. Charge Carrier Recombination Channels in the LowTemperature Phase of Organic-Inorganic Lead Halide Perovskite Thin Films. APL Mater. 2014, 2, 081513.

(33) Xing, G.; Mathews, N.; Sun, S.; Lim, S. S.; Lam, Y. M.; Grätzel, M.; Mhaisalkar, S.; Sum, T. C. Long-Range Balanced Electron- and Hole-Transport Lengths in Organic-Inorganic $\mathrm{CH}_{3} \mathrm{NH}_{3} \mathrm{PbI}_{3}$. Science 2013, 342, 344-347.

(34) Stamplecoskie, K. G.; Manser, J. S.; Kamat, P. V. Dual Nature of the Excited State in Organic-inorganic Lead Halide Perovskites. Energy Environ. Sci. 2015, 8, 208-215.

(35) Christians, J. A.; Manser, J. S.; Kamat, P. V. The Multifaceted Excited State of $\mathrm{CH}_{3} \mathrm{NH}_{3} \mathrm{PbI}_{3}$. Charge Separation, Recombination, and Trapping. J. Phys. Chem. Lett. 2015, 6, 2086-2095.
(36) Even, J.; Pedesseau, L.; Katan, C. Analysis of Multivalley and Multibandgap Absorption and Enhancement of Free Carriers Related to Exciton Screening in Hybrid Perovskites. J. Phys. Chem. C 2014, $118,11566-11572$.

(37) Wang, L.; Mccleese, C.; Kovalsky, A.; Zhao, Y.; Burda, C. Femtosecond Time-Resolved Transient Absorption Spectroscopy of $\mathrm{CH}_{3} \mathrm{NH}_{3} \mathrm{PbI}_{3}$ Perovskite Films: Evidence for Passivation Effect of $\mathrm{PbI}_{2}$. J. Am. Chem. Soc. 2014, 136, 12205-12208.

(38) Yoshida, H.; Ohno, N.; Fujita, M. Exciton Transition of $\mathrm{SnI}_{2}$ at the Fundamental Absorption Edge. Phys. Status Solidi B 1997, 203, 281-286.

(39) Even, J.; Pedesseau, L.; Katan, C.; Kepenekian, M.; Lauret, J.-S.; Sapori, D.; Deleporte, E. Solid State Physics Perspective on Hybrid Perovskite Semiconductors. J. Phys. Chem. C 2015, 119, 10161-10177.

(40) Even, J.; Pedesseau, L.; Jancu, J.-M.; Katan, C. DFT and K.p Modelling of the Phase Transitions of Lead and Tin Halide Perovskites for Photovoltaic Cells. Phys. Status Solidi RRL 2014, 8, 31-35.

(41) Fluegel, B.; Francoeur, S.; Mascarenhas, A.; Tixier, S.; Young, E. C.; Tiedje, T. Giant Spin-Orbit Bowing in GaAs ${ }_{1-\mathrm{x}} \mathrm{Bi}_{\mathrm{x}}$. Phys. Rev. Lett. 2006, 97, 067205.

(42) Fluegel, B.; Mascarenhas, A.; Ptak, A. J.; Tixier, S.; Young, E. C.; Tiedje, T. E+ Transition in $\mathrm{GaAs}_{1-\mathrm{x}} \mathrm{N}_{\mathrm{x}}$ and $\mathrm{GaAs}_{1-\mathrm{x}} \mathrm{Bi}_{\mathrm{x}}$ due to Isoelectronic-Impurity-Induced Perturbation of the Conduction Band. Phys. Rev. B: Condens. Matter Mater. Phys. 2007, 76, 155209.

(43) Wehrenfennig, C.; Liu, M.; Snaith, H. J.; Johnston, M. B.; Herz, L. M. Homogeneous Emission Line Broadening in the Organo Lead Halide Perovskite $\mathrm{CH}_{3} \mathrm{NH}_{3} \mathrm{PbI}_{(3-\mathrm{x})} \mathrm{Cl}_{\mathrm{x}}$. J. Phys. Chem. Lett. 2014, 5, 1300-1306.

(44) Shum, K.; Chen, Z.; Qureshi, J.; Yu, C.; Wang, J. J.; Pfenninger, W.; Vockic, N.; Midgley, J.; Kenney, J. T. Synthesis and Characterization of $\mathrm{CsSnI}_{3}$ Thin Films. Appl. Phys. Lett. 2010, 96, 221903.

(45) Chen, Y.; Kothiyal, G. P.; Singh, J.; Bhattacharya, P. K. Absorption and Photoluminescence Studies of the Temperature Dependence of Exciton Life Time in Lattice-Matched and Strained Quantum Well Systems. Superlattices Microstruct. 1987, 3, 657-664.

(46) Siebentritt, S.; Rey, G.; Finger, A.; Regesch, D.; Sendler, J.; Weiss, T. P.; Bertram, T. What Is the Bandgap of Kesterite? Sol. Energy Mater. Sol. Cells 2015, DOI: 10.1016/j.solmat.2015.10.017.

(47) Gokmen, T.; Gunawan, O.; Todorov, T. K.; Mitzi, D. B. Band Tailing and Efficiency Limitation in Kesterite Solar Cells. Appl. Phys. Lett. 2013, 103, 103506.

(48) Stranks, S. D.; Snaith, H. J. Metal-Halide Perovskites for Photovoltaic and Light-Emitting Devices. Nat. Nanotechnol. 2015, 10, 391-402.

(49) Johnston, M. B.; Herz, L. M. Hybrid Perovskites for Photovoltaics: Charge-Carrier Recombination, Diffusion, and Radiative Efficiencies. Acc. Chem. Res. 2016, 49, 146-154. 